\title{
Electrostatic cumulation of high-current electron beams for terahertz sources
}

\author{
S. V. Anishchenko, ${ }^{*}$ V. G. Baryshevsky, ${ }^{\dagger}$ and A. A. Gurinovich \\ Research Institute for Nuclear Problems Bobruiskaya street, 11, 220030, Minsk, Belarus
}

(Received 3 December 2018; published 18 April 2019)

\begin{abstract}
The electrostatic cumulation of current density in relativistic vacuum diodes with ring-type cathodes is described theoretically and confirmed experimentally. The distinctive feature of the suggested cumulation mechanism is a very low energy spread of electrons. As a result of electrostatic cumulation, a thin relativistic electron beam with a current density of $1 \mathrm{kA} / \mathrm{mm}^{2}$ can be formed. This quantity exceeds typical current density values in high-current Cherenkov sources by an order of magnitude. Such a beam can be used as an active medium in high-power terahertz sources.
\end{abstract}

DOI: 10.1103/PhysRevAccelBeams.22.043403

\section{INTRODUCTION}

Generation of high-power radiation is one of the main goals of vacuum terahertz electronics [1,2]. The progress in this field is strongly connected with production in highcurrent accelerators [1-3] of relativistic electron beams with ever-increasing density.

A typical high-current accelerator for a $\mathrm{THz}$ source consists of an axially symmetric cathode and anode [3]. The cathode is hemispherical with curvature radius of several centimeters and it is spaced tens of millimeters from the concave anode. The accelerator produces about $1 \mathrm{kA}$ of total current at $\sim 1 \mathrm{MV}$ applied voltage. The hole in the anode cuts a small central part of the accelerated beam ( $\sim 10 \mathrm{~A})$, which is guided by magnetic field with the strength $\sim 1 \mathrm{~T}$. Low efficiency of beam usage limits applications of such an approach.

The second disadvantage of applying traditional highcurrent accelerators is high value of an external guiding field $(1 \mathrm{~T})$ that results in large dimensions of the whole system. High magnetic fields are necessary to prevent beam expansion due to the Coulomb repulsion. Meanwhile an alternative mechanism precluding beam expansion is already well-known. This mechanism does not require external guiding fields. It is based on magnetic cumulation discovered by Bennett [4]. Magnetic cumulation provides charged-particle beam intensities as high as $10 \mathrm{GW} / \mathrm{mm}^{2}$ $[5,6]$, thus enabling the laboratory investigation of the

\footnotetext{
*sanishchenko@mail.ru

tbar@inp.bsu.by

gur@inp.bsu.by
}

Published by the American Physical Society under the terms of the Creative Commons Attribution 4.0 International license. Further distribution of this work must maintain attribution to the author(s) and the published article's title, journal citation, and DOI. extreme state of matter. However, the large particle energy spread does not enable to use magnetically cumulated electron beams in terahertz radiation sources.

This paper considers one more alternative for highcurrent electron beam cumulation, namely electrostatic cumulation $[7,8]$. Occurring in a relativistic vacuum diode with a ring-type cathode, this mechanism does not suffer from particle energy spread [8]. Our principle task is to provide theoretical description of cumulation mechanism and the experimental verification thereof. We will show that electrostatic cumulation provides current densities $\left(\sim 1 \mathrm{kA} / \mathrm{mm}^{2}\right)$ sufficient to seed high-power terahertz radiation sources.

\section{THE PHENOMENON OF ELECTROSTATIC CUMULATION}

Electrostatic cumulation was first revealed during modeling of high-current accelerators [8]. The qualitative picture of electrostatic cumulation can be described as follows. In a relativistic vacuum diode, electron emission is most intense from the cathode's edges (Fig. 1). Let us consider electrons emitted from the inner edge. The Coulomb repulsion causes the charged particles to rush to the region free from the beam. As a result, the accelerated motion of electrons toward the anode comes alongside the radial motion to the cathode's symmetry axis. As a result, the high-current beam density increases multifold on the axis as compared to the average current density in the cathode-anode gap $[7,8]$.

Figure 2 shows the results of simulations: the dose absorbed by the anode. The assumed parameters of the cathode are as follows: cathode radius $30 \mathrm{~mm}$, cathodeanode gap $16 \mathrm{~mm}$, and the radius of the inner hole $10 \mathrm{~mm}$. The maximum value of the accelerating voltage pulse is taken equal to $360 \mathrm{kV}$ and its duration is $130 \mathrm{~ns}$. The simulated current density in the region of the central spot on 


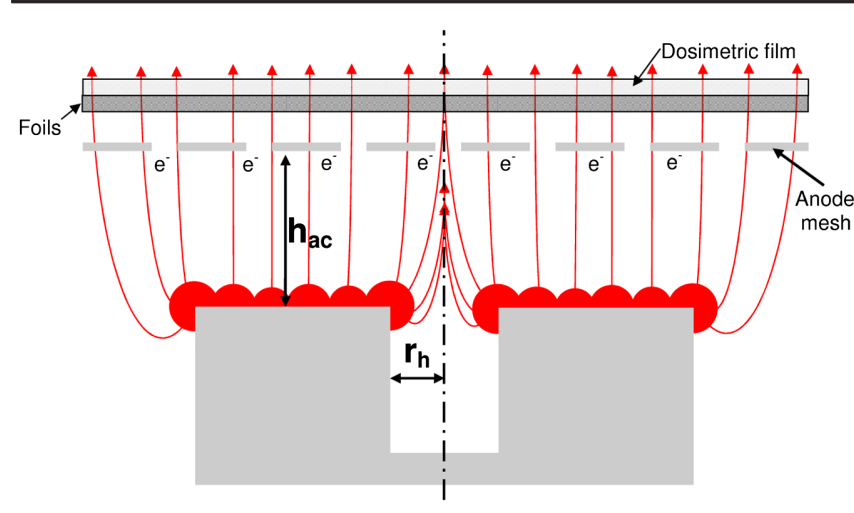

FIG. 1. Cumulation mechanism.

the anode at the moment corresponding to the maximum accelerating voltage is as large as $10 \mathrm{~A} / \mathrm{mm}^{2}$, being 5 times greater than the average current density of the high-current diode. A typical radius of the spot is about $1 \mathrm{~mm}$. Thus, the simulation result indicates the electron-beam cumulation on the axis of a high-current diode with a ring-type cathode.

The undeniable advantage of electrostatic cumulation is a very low energy spread of particles due to the stationary flow of charged particles. In contrast, self-focusing of a beam by its own magnetic field leads to a turbulent flow, and the charged particles acquire a significant energy spread. In this case, the electron flow is like a compressed relativistic gas with the electron temperature $T_{e}$ determined by the accelerating voltage, $T_{e} \sim \frac{q_{e} U}{k_{B}}$. (Symbol $k_{B}$ denotes the Boltzman constant.)

To obtain information about electron beam parameters, we use a nanosecond pulse-periodic electron accelerator with a compact SF6-insulated high-voltage generator providing pulsed voltage up to $400 \mathrm{kV}$ in $30 \mathrm{Ohm}$ resistive

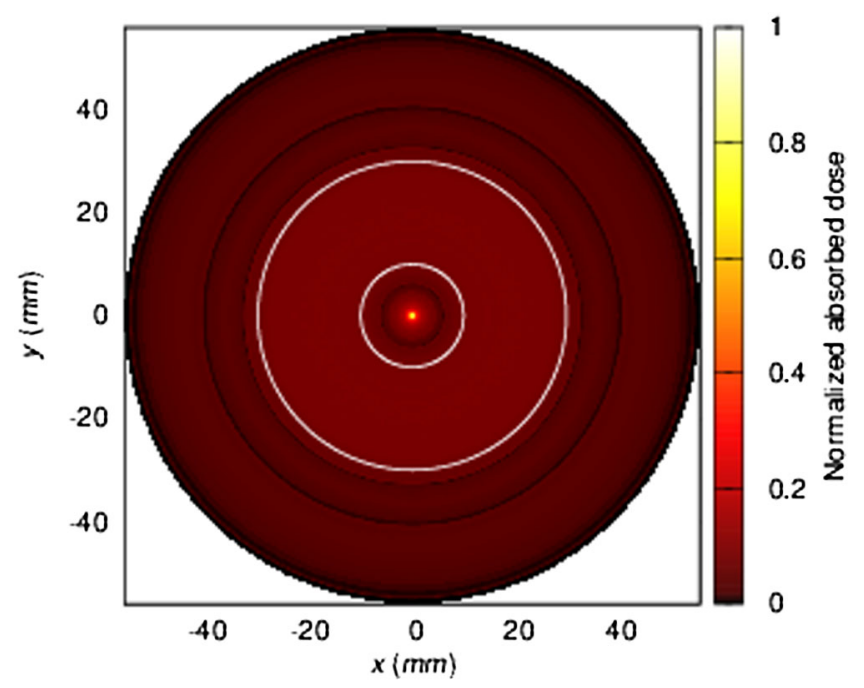

FIG. 2. Electrostatic cumulation: simulation results [cathode radius $r_{c}=30 \mathrm{~mm}$, anode-cathode gap $h_{a c}=16 \mathrm{~mm}$, hole radius $r_{h}=10 \mathrm{~mm}$.

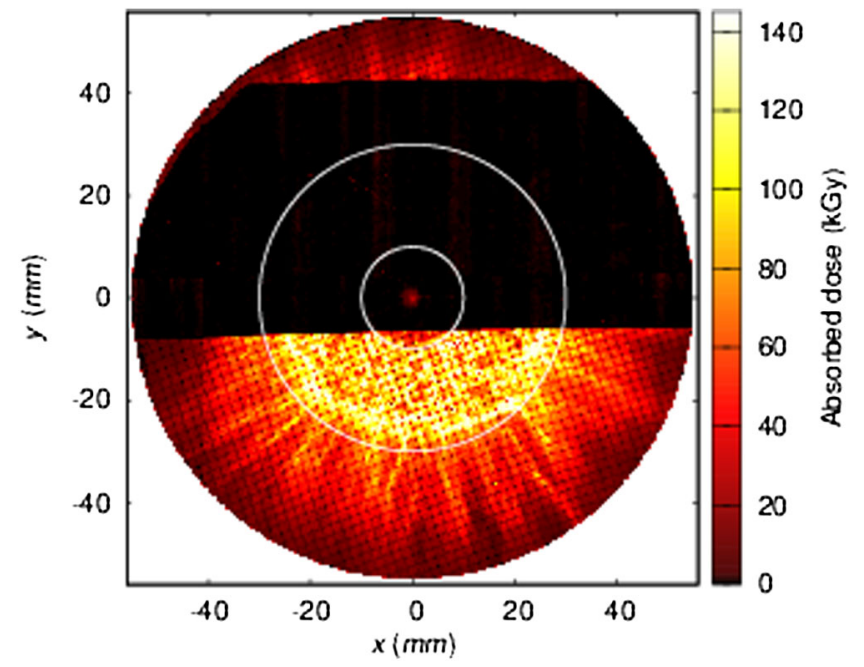

FIG. 3. Electrostatic cumulation: experimental results [cathode radius $r_{c}=30 \mathrm{~mm}$, anode-cathode gap $h_{a c}=16 \mathrm{~mm}$, hole radius $\left.r_{h}=10 \mathrm{~mm}\right]$.

load with a full width at half maximum of $130 \mathrm{~ns}$ and rise time of $30 \mathrm{~ns}$ [7]. To obtain full-sized imprints of electron beams, we use a radiochromic dosimetry film placed $3 \mathrm{~mm}$ behind the anode mesh made of stainless steel (the geometrical transparency of the mesh is 0.77); the cathode-anode gap is $20 \mathrm{~mm}$. The dosimetry film enables to obtain information about the total absorbed dose over the beam cross section, caused by passage of charged particles.

Our first experiments had shown that the intense flow of charged particles on the axis had burned the film through. For this reason, in further experiments we placed $70 \mu \mathrm{m}$ thick aluminium foil in front of the dosimetry film to decrease the absorbed dose (see Fig. 3) [7]. This enables us to cut off the flows of both the cathode plasma and the weakly-relativistic electrons produced at the voltage pulse decay. The experiments conducted with one, two, and three foils demonstrated that a sharp increase in the absorbed dose remains in the center. This means that the particle flow consists of high-energy electrons at the beam axis. In the experiments with three foil layers cutting off all electrons whose energy was less than $250 \mathrm{keV}$, the absorbed dose in the center was almost four times as large as the average dose across the beam cross section, showing a good agreement with the simulation results.

\section{TRANSPORT OF A DENSE ELECTRON BEAM AT 4 MEV}

Let us note here that both the simulations and the experiments were performed at maximum accelerating voltage $400 \mathrm{kV}$. The estimates show that it is possible to achieve the beam current density more than $1 \mathrm{kA} / \mathrm{mm}^{2}$ with accelerating voltages of several megavolts. This quantity is several times higher than those obtained in existing high-current terahertz sources producing $100 \mathrm{~kW}$ 


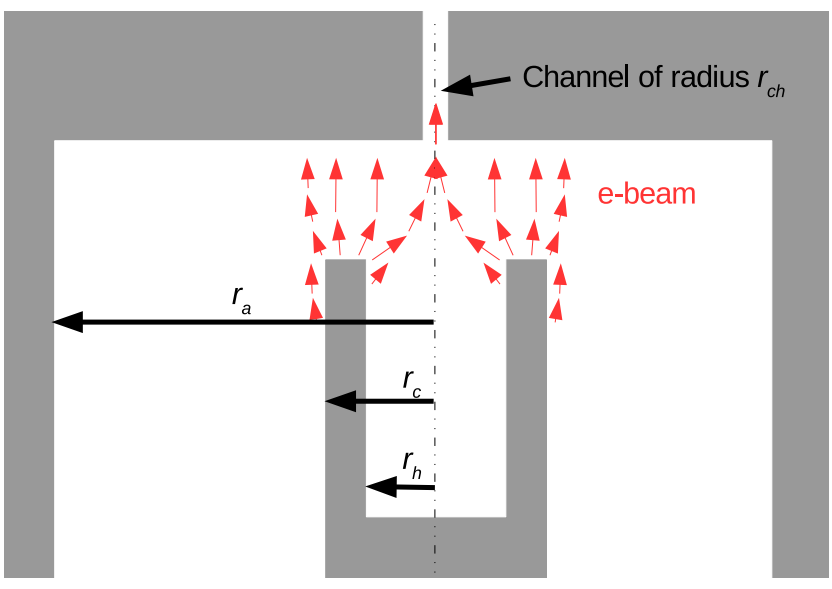

FIG. 4. Extraction of cumulated electron beams.

of radiation power. The cumulated electron beam can be extracted with the help of a thin channel in the anode (Fig. 4). The beam may be used in terahertz sources.

Cumulated electron beams are explored with the particlein-cell code described in Appendix. The accelerator geometry consists of two parts: a cathode and an anode with a channel in its center. The cathode has an outer radius $r_{c}$ of $12 \mathrm{~mm}$. The hole radius $r_{h}$ has a value $10 \mathrm{~mm}$. The cathodeanode gap $h_{a c}$ is $16 \mathrm{~mm}$. The channel radius $r_{c h}$ has values 0.5 or $1 \mathrm{~mm}$. The anode radius $r_{a}$ is $150 \mathrm{~mm}$.

Simulations show that a typical current density in the channel $j_{a v}$ is about $1 \mathrm{kA} / \mathrm{mm}^{2}$ (see Fig. 5). Trajectories of sample particles emitted from the inner edge of the cathode are shown on Fig. 6. The particles are accelerated by the electric field depicted on Figs. 7 and 8. The radial component of the electric field gives a strong initial inward kick to the electrons. This is the reason for electrostatic cumulation. Then the accelerated particles are accelerated toward the anode and cross the axis (see Fig. 6).

A typical distribution of current density is shown on Fig. 9. The simulated distribution is likely invalid in the region comparable to the spatial dimension of the grid cells,

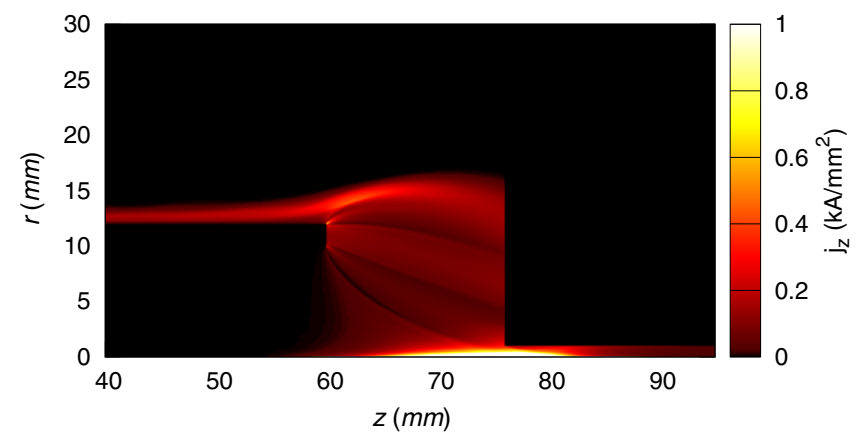

FIG. 5. Sample of simulated current density at $4 \mathrm{MV}$ of applied voltage [channel radius $r_{\mathrm{ch}}=1 \mathrm{~mm}$, channel entrance $\left.z_{\mathrm{ch}}=76 \mathrm{~mm}\right]$.

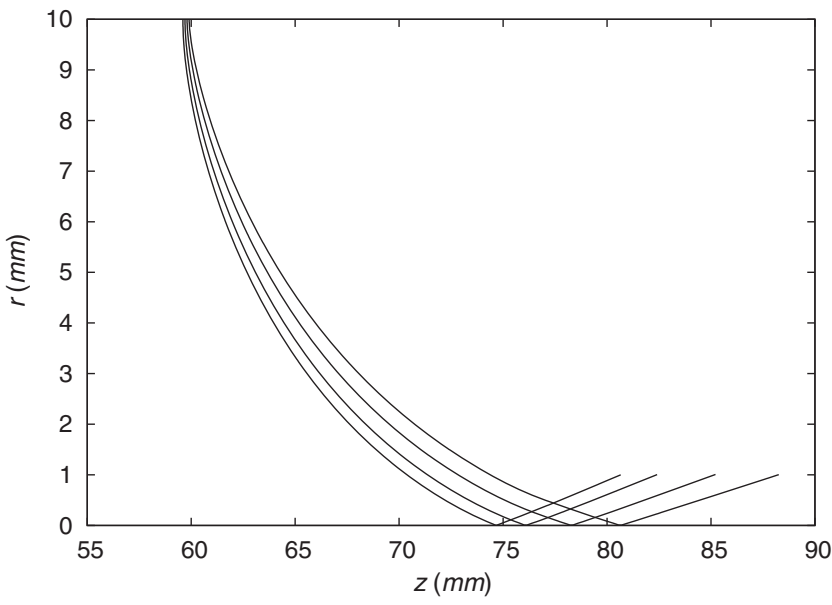

FIG. 6. Trajectories of sample particles emitted from the inner edge of the cathode.

$\Delta R=0.1 \mathrm{~mm}$, used in simulations. However, at larger $r$, we can assume $j_{z} r \approx 0.35 \mathrm{kA} / \mathrm{mm}$.

Now, let us say a few words about parasitic beam instabilities that may arise in the channel at high current densities, $j \sim 1 \mathrm{kA} / \mathrm{mm}^{2}$. At $4 \mathrm{MeV}$, a typical plasma frequency in the channel is $f_{\mathrm{pl}} \approx 15 \mathrm{GHz}$. The quantity is much less than radiation terahertz frequencies and the critical wavelength of the channel $f_{\text {crit }} \approx 100 \mathrm{GHz}$. Moreover, the transport distance and channel radius is

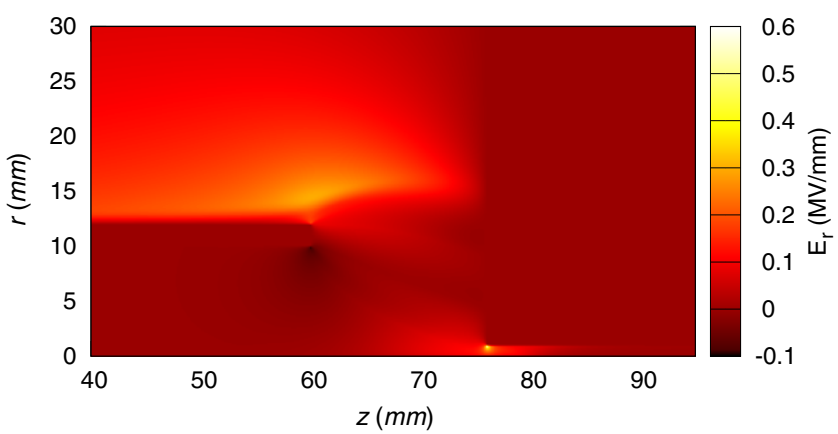

FIG. 7. Radial component of electric field strength.

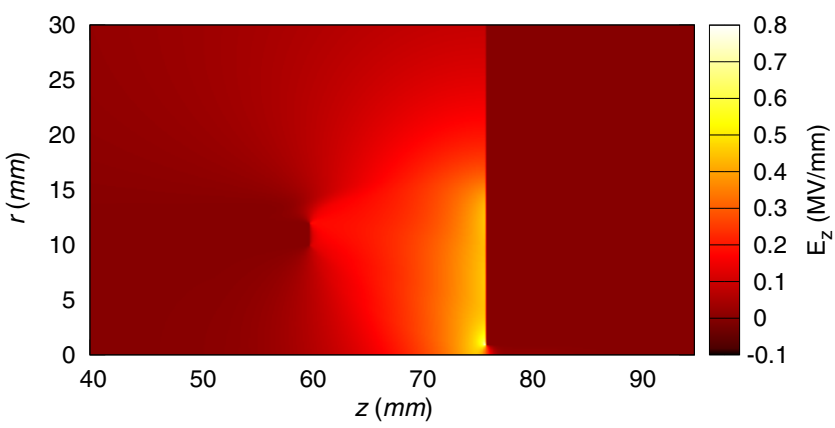

FIG. 8. Longitudinal component of electric field strength. 


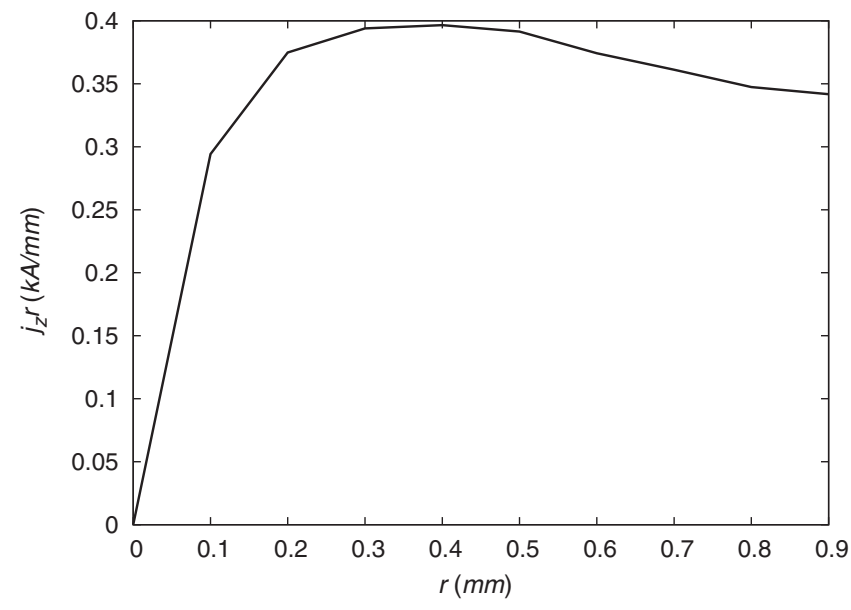

FIG. 9. Current density at channel entrance.

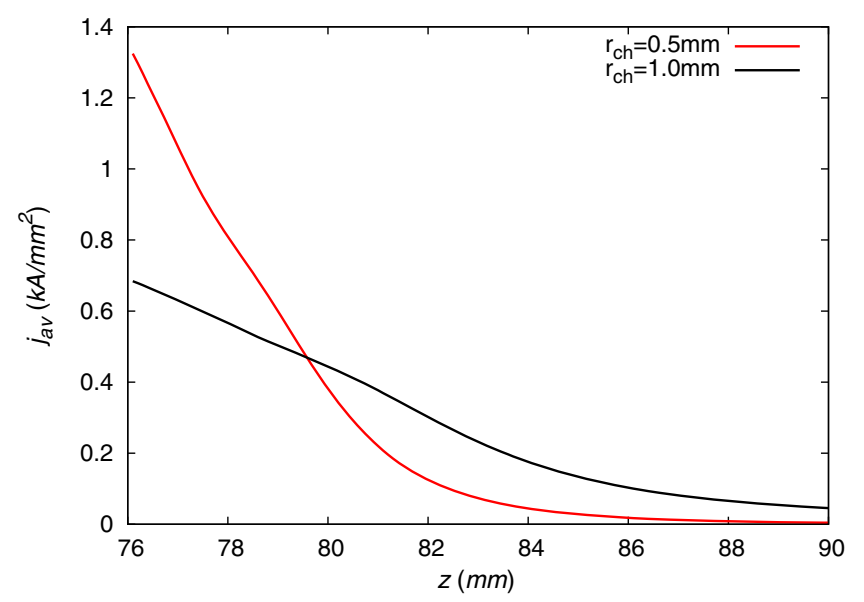

FIG. 10. Average current density as a function of transport distance [channel entrance $z_{\mathrm{ch}}=76 \mathrm{~mm}$ ].

sufficiently less than the plasma wavelength $\lambda_{\mathrm{pl}} \sim c f_{\mathrm{pl}} \approx$ $20 \mathrm{~mm}$. That is why parasitic beam instabilities have not enough time and space to develop.

The transport length of cumulated beam lies in the millimeter range (Fig. 10). Estimations show that the cumulated electron beam, injected in the slow-wave structure, can lead to the Cherenkov terahertz radiation of 40-400 MW power if we assume 1\%-10\% efficiency, which is a typical value for the high-current Cherenkov generators $[3,9]$.

\section{CONCLUSIONS}

In this paper we describe a new cumulation mechanism for high-current beams in relativistic vacuum diodes with a ring-type cathode. The basis of the cumulation mechanism is electrostatic repulsion of electrons emitted from the inner edge of the cathode. At several hundred kilovolts, the electrostatic cumulation is verified experimentally. The current density and beam intensity equal to
1-10 GW/ $\mathrm{mm}^{2}$ and $1 \mathrm{kA} / \mathrm{mm}^{2}$, respectively, are obtained in simulations at $4 \mathrm{MV}$.

A very low particle energy spread in the region of maximum current density is a distinctive feature of the described cumulation mechanism. Due to electrostatic cumulation, a thin relativistic electron flow with a radial width of $1 \mathrm{~mm}$ and beam current density of $1 \mathrm{kA} / \mathrm{mm}^{2}$ is formed. This quantity is several times higher than that obtained in existing high-current terahertz sources producing 0.1-1 MW of radiated power. As a result, electrostatic cumulation can sufficiently increase the radiation output. One of the main advantages of high-current terahertz sources with electrostatic cumulation is the absence of external magnetic fields causing large dimensions of the whole system.

\section{APPENDIX: SIMULATION OF HIGH-CURRENT ELECTRON BEAMS}

Self-consistent simulation of relativistic motion in highcurrent diodes is usually performed using the particle-incell method [10-16]. The method is often implemented in a quasistationary approximation $[10,11]$. The approximation is applied when electric and magnetic fields are slowly varying functions of time. As a result, the displacement currents and induction fields can be neglected.

As we are going to deal with axially symmetric geometries the simulation is performed in cylindrical coordinates. So, let us introduce the space mesh as follows:

$$
\begin{aligned}
\omega_{\mathrm{RZ}} & =\left\{R_{i}=i \Delta R, R_{j}=j \Delta Z,\right. \\
i & \left.=0,1, \ldots, i_{\max }, j=0,1, \ldots, j_{\max }\right\}
\end{aligned}
$$

and the time mesh

$$
\omega_{T}=\left\{T_{n}=n \Delta T, n=0,1, \ldots\right\} .
$$

Spatial dimensions of the grid cells are set to be equal, i.e., $\Delta R=\Delta Z$.

The method implies representation of charged particles' flow as a collection of macroparticles. Each of them contains a large number of real charge carriers. Macroparticles have certain spatial distributions of mass and charge those contribute to the physical quantities (such as current and charge densities) defined on the grid. The latter give us possibility to find electric and magnetic fields in the grid nodes. Interpolation of the grid fields to macroparticles' location determines forces acting on charges. As a result, new particles' locations and velocities can be found. At this point, all the procedures are repeated again.

Now, let us proceed to the brief description of the algorithm used in our simulations. 
Electric field $\vec{E}$ is expressed by electrostatic potential $\phi$,

$$
\vec{E}=-\nabla \phi
$$

The potential $\phi$ obeys the Poisson equation,

$$
\Delta \phi=-4 \pi \rho .
$$

Symbol $\rho$ denotes the charge density.

To solve the Poisson equation, we use the Jacobi iterative method [13],

$$
\begin{aligned}
\frac{\phi_{i+1, j}^{n, s}(1+0.5 / i)-2 \phi_{i, j}^{n, s+1}+\phi_{i-1, j}^{n, s}(1-0.5 / i)}{\Delta R^{2}}+\frac{\phi_{i, j+1}^{n, s}-2 \phi_{i, j}^{n, s+1}+\phi_{i, j-1}^{n, s}}{\Delta Z^{2}}=-4 \pi \rho_{i, j}^{n} & \text { for } i \neq 0, \\
\frac{8 \phi_{i+1, j}^{n, s}-8 \phi_{i, j}^{n, s+1}}{\Delta R^{2}}+\frac{\phi_{i, j+1}^{n, s}-2 \phi_{i, j}^{n, s+1}+\phi_{i, j-1}^{n, s}}{\Delta Z^{2}}=-4 \pi \rho_{i, j}^{n} \quad & \text { for } i=0, \\
\phi_{i, j}^{n, 0}=\phi_{i, j}^{n-1}, &
\end{aligned}
$$

proven to be effective in plasma dynamics and for solving the vacuum electronic problems [8]. Here, $s$ is the iteration number and $n$ is the time step number. Iterations in (A6) occur until all the quantities $\left|\phi^{n, s}-\phi^{n, s-1}\right|$ become less than $\epsilon U$. The parameter $\epsilon \ll 1$ is a small fraction of accelerating voltage $U$. (In our simulations, $\epsilon$ is set to be equal to $10^{-5}$.)

As a rule, iterative methods are slowly converging [13]. However, this is not always the case in dynamical problems [8]. The main reason is proper choice of initial approximation in the iteration scheme. Namely, the potential distribution at the previous time step $n-1$ plays the role of initial approximation at the step $n$. As a result, the Jacobi method reduces to one-three iterations at each time step. The procedure requires much less simulation time than, say, the computation of new velocities and positions of the particles.

To solve the Poisson equation, we use the Dirichlet boundary conditions. The conditions imply specification of potentials at the cathode $\left(\phi=U_{c}\right)$ and the anode $\left(\phi=U_{a}\right)$ and at the edge of the computational region $(z=0)$. For the latter, the logarithmic distribution is used [9]

$$
\phi=U_{c}+\frac{\left(U_{a}-U_{c}\right) \ln \left(r / r_{c}\right)}{\ln \left(r_{a} / r_{c}\right)}
$$

This distribution exactly describes $\phi$ change in the gap between two infinite cylinders. The potential distribution approaches (A5) if the edge of the computational region $(z=0)$ is located at a considerable distance from the electron-emitting surface.

Charge density $\rho_{i, j}^{n}$ can be found with the linear weighting procedure [14]. This means that each particle contributes to the charge density of the four nearest nodes according to the following formulas

$$
\begin{aligned}
\rho_{i, j}^{n} & =\sum_{\alpha} \Delta \rho_{i, j}^{\alpha, n}, \\
\Delta \rho_{i, j}^{\alpha n} & =\frac{q_{\alpha}}{\Delta V}\left[1-\frac{\left(r_{\alpha}^{n}\right)^{2}-(\Delta R i)^{2}}{\Delta R^{2}(2 i+1)}\right]\left(1-\frac{z_{\alpha}^{n}-\Delta Z j}{\Delta Z}\right), \\
\Delta \rho_{i+1, j}^{\alpha n} & =\frac{q_{\alpha}}{\Delta V} \frac{\left(r_{\alpha}^{n}\right)^{2}-(\Delta R i)^{2}}{\Delta R^{2}(2 i+1)}\left(1-\frac{z_{\alpha}^{n}-\Delta Z j}{\Delta Z}\right), \\
\Delta \rho_{i, j+1}^{\alpha n} & =\frac{q_{\alpha}}{\Delta V}\left[1-\frac{\left(r_{\alpha}^{n}\right)^{2}-(\Delta R i)^{2}}{\Delta R^{2}(2 i+1)}\right] \frac{z_{\alpha}^{n}-\Delta Z j}{\Delta Z}, \\
\Delta \rho_{i+1, j+1}^{\alpha n} & =\frac{q_{\alpha}}{\Delta V} \frac{\left(r_{\alpha}^{n}\right)^{2}-(\Delta R i)^{2}}{\Delta R^{2}(2 i+1)} \frac{z_{\alpha}^{n}-\Delta Z j}{\Delta Z} .
\end{aligned}
$$

Here,

$$
\begin{aligned}
& \Delta V=\pi \Delta R^{2} \Delta Z(2 j+1) \quad \text { for } i \neq 0, \\
& \Delta V=\frac{\pi \Delta R^{2} \Delta Z}{4} \quad \text { for } i=0
\end{aligned}
$$

$q_{\alpha}$ and $\vec{r}_{\alpha}^{n}=\left(r_{\alpha}^{n}, z_{\alpha}^{n}\right)$ are particles' charges and positions, respectively.

The electric field is related to electrostatic potential $\phi_{i, j}^{n}$ as

$\vec{E}_{i, j}^{n}=-\left(\frac{\phi_{i+1, j}^{n}-\phi_{i-1, j}^{n}}{2 \Delta R}, \frac{\phi_{i, j+1}^{n}-\phi_{i, j-1}^{n}}{2 \Delta Z}\right)$ for $i \neq 0$,

$\vec{E}_{i, j}^{n}=-\left(0, \frac{\phi_{i, j+1}^{n}-\phi_{i, j-1}^{n}}{2 \Delta Z}\right) \quad$ for $i=0$.

A nonrotating axially symmetric electron beam possesses only the polar component of the self-induced 
magnetic field $H$. The component $H$ can be found using the Stokes theorem:

$$
H=\frac{4 \pi \int_{0}^{r} j_{z}\left(r_{1}, z\right) r_{1} d r_{1}}{c r} .
$$

(To calculate the magnetic field properly, one must include the current in the cathode itself $[9,10]$.)

Note that the axial component of the current density can be found by the same weighting procedure as the charge density. It is only necessary to substitute $q_{\alpha} v_{z \alpha}^{n}$ instead of $q_{\alpha}$ in (A6).

Numerical integration of the Newton-Lorentz equations is the most time-consuming procedure. That is why the integration algorithm must be as fast as possible. During several decades the Boris algorithm was used for this purpose. However, this algorithm can lead to spurious forces acting on particles [17]. To overcome the drawback, a new algorithm for momenta integration has been proposed by J.-L. Vay. In the axially symmetric case, the numerical scheme can be written as follows

$$
\begin{aligned}
p_{r \alpha}^{n+1 / 2} & =p_{r \alpha}^{n}+q_{\alpha}\left(E_{r \alpha}^{n+1 / 2}-\frac{v_{z \alpha}^{n}}{2 c} H_{\alpha}^{n+1 / 2}\right) \Delta T, \\
p_{z \alpha}^{n+1 / 2} & =p_{z \alpha}^{n}+q_{\alpha}\left(E_{z \alpha}^{n+1 / 2}+\frac{v_{r \alpha}^{n}}{2 c} H_{\alpha}^{n+1 / 2}\right) \Delta T, \\
\gamma_{\alpha}^{n+1} & =\sqrt{1+\frac{\left(p_{r \alpha}^{n}+q_{\alpha} E_{r \alpha}^{n+1 / 2} \Delta T\right)^{2}}{m_{\alpha}^{2} c^{2}}+\frac{\left(p_{z \alpha}^{n}+q_{\alpha} E_{z \alpha}^{n+1 / 2} \Delta T\right)^{2}}{m_{\alpha}^{2} c^{2}}}, \\
A_{\alpha} & =\frac{q_{\alpha} H_{\alpha}^{n} \Delta T}{2 m_{\alpha} c \gamma_{\alpha}^{n+1}}, \\
B_{\alpha} & =1+A_{\alpha}^{2}, \\
p_{r \alpha}^{n+1} & =\left(p_{r \alpha}^{n+1 / 2}-A_{\alpha} p_{z \alpha}^{n+1 / 2}\right) / B_{\alpha}, \\
p_{z \alpha}^{n+1} & =\left(p_{z \alpha}^{n+1 / 2}+A_{\alpha} p_{r \alpha}^{n+1 / 2}\right) / B_{\alpha}, \\
v_{r \alpha}^{n+1} & =\frac{p_{r \alpha}^{n+1}}{\gamma_{\alpha}^{n+1} m_{\alpha}}, \\
v_{z \alpha}^{n+1} & =\frac{p_{z \alpha}^{n+1}}{\gamma_{\alpha}^{n+1} m_{\alpha}} .
\end{aligned}
$$

Here, $E_{r \alpha}^{n+1 / 2}, E_{z \alpha}^{n+1 / 2}$, and $H_{\alpha}^{n+1 / 2}$ are the electric and magnetic fields acting on a particle with momenta $\vec{p}_{\alpha}$ and velocity $\vec{v}_{\alpha}$ at time $\Delta T(n+1 / 2)$.

In quasistationary flows, the fields are slowly varying functions of time. This property gives us the possibility to use $E_{r \alpha}^{n}, E_{z \alpha}^{n}$, and $E_{z \alpha}^{n}$ instead of $E_{r \alpha}^{n+1 / 2}, E_{z \alpha}^{n+1 / 2}$, and $H_{\alpha}^{n+1 / 2}$, respectively [10]. The former quantities can be found by weighting procedure [14], for example,

$$
\begin{aligned}
H_{\alpha}^{n}= & H_{i, j}\left[1-\frac{\left(r_{\alpha}^{n}\right)^{2}-(\Delta R i)^{2}}{\Delta R^{2}(2 i+1)}\right]\left(1-\frac{z_{\alpha}^{n}-\Delta Z j}{\Delta Z}\right), \\
& +H_{i+1, j} \frac{\left(r_{\alpha}^{n}\right)^{2}-(\Delta R i)^{2}}{\Delta R^{2}(2 i+1)}\left(1-\frac{z_{\alpha}^{n}-\Delta Z j}{\Delta Z}\right), \\
& +H_{i, j+1}\left[1-\frac{\left(r_{\alpha}^{n}\right)^{2}-(\Delta R i)^{2}}{\Delta R^{2}(2 i+1)}\right] \frac{z_{\alpha}^{n}-\Delta Z j}{\Delta Z}, \\
& +H_{i+1, j+1} \frac{\left(r_{\alpha}^{n}\right)^{2}-(\Delta R i)^{2}}{\Delta R^{2}(2 i+1)} \frac{z_{\alpha}^{n}-\Delta Z j}{\Delta Z}
\end{aligned}
$$

where magnetic field $H$ is calculated in four nearest to the particle nodes.

Using central difference approximations for velocities,

$$
\begin{aligned}
& v_{r \alpha}^{n+1 / 2}=\frac{1}{2}\left(v_{r \alpha}^{n}+v_{r \alpha}^{n+1}\right), \\
& v_{z \alpha}^{n+1 / 2}=\frac{1}{2}\left(v_{z \alpha}^{n}+v_{z \alpha}^{n+1}\right),
\end{aligned}
$$

we update the positions [10],

$$
\begin{aligned}
& r_{\alpha}^{n+1}=r_{\alpha}^{n}+v_{r \alpha}^{n+1 / 2} \Delta T, \\
& z_{\alpha}^{n+1}=z_{\alpha}^{n}+v_{z \alpha}^{n+1 / 2} \Delta T .
\end{aligned}
$$

If $r_{\alpha}^{n+1}$ becomes negative then $r_{\alpha}^{n+1}$ and $v_{r \alpha}^{n+1}$ change sign,

$$
\begin{aligned}
& r_{\alpha}^{n+1} \rightarrow-r_{\alpha}^{n+1} \\
& v_{r \alpha}^{n+1} \rightarrow-v_{r \alpha}^{n+1} .
\end{aligned}
$$

Two types of particle injection are implemented in the simulation code, namely, overinjection and the dual cell algorithm described in [18]. They both lead to the similar results.

In simulations, a simple uniform grid with $\Delta R=\Delta Z=$ $100 \mu \mathrm{m}$ is used. The time step $\Delta T$ is set to be equal to $0.5 \Delta Z / c \approx 167 \mathrm{fs}$.

[1] J.H. Booske, Vacuum electronic high power terahertz sources, IEEE Trans. Terahertz Sci. Technol. 1, 54 (2011).

[2] S.S. Dhillon et al., The 2017 terahertz science and technology roadmap, J. Phys. D 50, 043001 (2017).

[3] E. P. Garate, J. Walsh, C. Shaughnessy, B. Johnson, and S. Moustaizis, Cherenkov free electron laser operation from 375 to $1000 \mu \mathrm{m}$, Nucl. Instrum. Methods Phys. Res., Sect. A 259, 125 (1987).

[4] W. H. Bennett, Magnetically self-focussing streams, Phys. Rev. 45, 890 (1934).

[5] D. L. Morrow, J. Daniel Phillips, R. M. Stringeld, Jr., W. O. Doggett, and W. H. Bennett, Concentration and guidance of intense relativistic electron beams, Appl. Phys. Lett. 19, 441 (1971). 
[6] L. P. Bradley and G. W. Kuswa, Neutron Production and Collective Ion Acceleration in a High-Current Diode, Phys. Rev. Lett. 29, 1441 (1972).

[7] S. Anishchenko, V. Baryshevsky, N. Belous, A. Gurinovich, E. Gurinovich, E. Gurnevich, and P. Molchanov, Cumulation of high-current electron beams: Theory and experiment, IEEE Trans. Plasma Sci. 45, 2739 (2017).

[8] S. Anishchenko and A. Gurinovich, Simulation of explosive-emission and electron beam dynamics in a cathodeanode gap for cathode and anode optimization in an axial vircator, in Conference program of the 5th Euro-Asian Pulsed Power Conference (Kumamoto University, Kumamoto, 2014), Report No. OA1-5.

[9] S. P. Bugaev et al., Formation of high-current relativistic electron beams for high power microwave generators and ampliers, in High-Frequency Relativistic Electronics (Institute of Applied Physics, Gorky, 1979), pp. 5-75 [in Russian].

[10] J. W. Poukey, J. R. Freeman, and G. Yonas, Simulation of relativistic electron beam diodes, J. Vac. Sci. Technol. 10, 954 (1973).
[11] G. T. Golovin, Problems of the numerical simulation of stationary charged-particle beams, USSR Computational Mathematics and Mathematical Physics 29, 67 (1989).

[12] A. S. Roshal, Modeling of Charged Beams (Atomizdat, Moscow, 1979).

[13] R. W. Hockney and J. W. Easwood, Computer Simulation Using Particles (McGraw-Hill, New York, 1981).

[14] C. K. Birdsall and A. B. Langdon, Plasma Physics, via Computer Simulations (McGraw-Hill, New York, 1985).

[15] T. M. Antonsen, A. A. Mondelli, B. Levush, J. P. Verboncoeur, and C. K. Birdsall, Advances in modeling and simulation of vacuum electronic devices, Proc. IEEE 87, 804 (1999).

[16] J. P. Verboncoeur, Particle simulation of plasmas: review and advances, Plasma Phys. Controlled Fusion 47, A231 (2005).

[17] J.-L. Vay, Simulation of beams or plasmas crossing at relativistic velocity, Phys. Plasmas 15, 056701 (2008).

[18] J. J. Watrous, J. W. Lugisland, and G. E. Sasser, An improved space-charge-limited emission algorithm for use in particle-in-cell codes, Phys. Plasmas 8, 289 (2001). 